\title{
The 16th microlensing season of the OGLE survey
}

\author{
Andrzej Udalski* \\ Warsaw University Astronomical Observatory \\ Al. Ujazdowskie 4 \\ 00-478 Warszawa, Poland \\ E-mail: udalskieastrouw.edu.pl
}

\begin{abstract}
We present the most recent results from the 16th observational season of the Optical Gravitational Lensing Survey (OGLE) in 2007. History of the OGLE project since it beginnings in 1992 is presented. Among the main OGLE results are the detections of the microlensing events towards the Galactic Bulge and Magellanic Clouds, planetary microlensing events and continuous monitoring of strong lenses. Other research areas include also studies of variable stars and planetary transits. Future plans of the project include a new mosaic camera with 1.4 square degree field of view, to be in operation in early 2009.

Readers are referred to the attached on-line material which include full Power Point presentation and its PDF version.
\end{abstract}

The Manchester Microlensing Conference: The 12th International Conference and ANGLES Microlensing Workshop

January 21-25 2008

Manchester, $U K$

${ }^{*}$ Speaker. 